\title{
THE EVALUATION OF REDUNDANCY FOR ROAD TRAFFIC NETWORKS
}

\author{
Rawia Ahmed El-Rashidy ${ }^{1}$, Susan Grant-Muller ${ }^{2}$ \\ ${ }^{1}$ Institute of Railway Research, School of Computing and Engineering, \\ University of Huddersfield, United Kingdom \\ ${ }^{2}$ Institute for Transport Studies, University of Leeds, United Kingdom
}

Submitted 18 June 2014; resubmitted 7 January 2015; accepted 27 April 2015

\begin{abstract}
This paper presents two redundancy indices for road traffic network junctions and also an aggregated network redundancy index. The proposed redundancy indices could be implemented to identify optimal design alternatives during the planning stage of the network junctions whereas the aggregated network redundancy index could assess the best control and management policies under disruptive events. Furthermore, effective measures of network redundancy are important to policy makers in understanding the current resilience and future planning to mitigate the impacts of greenhouse gases. The proposed junction indices cover the static aspect of redundancy, i.e. alternative paths, and the dynamic feature of redundancy reflected by the availability of spare capacity under different network loading and service level. The proposed redundancy indices are based on the entropy concept, due to its ability to measure the system configuration in addition to being able to model the inherent uncertainty in road transport network conditions. Various system parameters based on different combinations of link flow, relative link spare capacity and Relative Link Speed (RLS) were examined. However, the two redundancy indices developed from the combined RLS and relative link spare capacity showed strong correlation with junction delay and volume capacity ratio of a synthetic road transport network of Delft city. Furthermore, the developed redundancy indices responded well to demand variation under the same network conditions and supply variations. Another case study on Junction 3A in M42 motorway near Birmingham demonstrated that the developed redundancy index is able to reflect the impact of the Active Traffic Management (ATM) scheme introduced in 2006.
\end{abstract}

Keywords: redundancy; road traffic networks; entropy; disruptive events; active traffic management.

\section{Introduction}

The importance of redundancy has been highlighted in many disciplines. For example, Downer (2009) argued that redundancy in technical systems should be understood as a 'design paradigm' as redundancy not only allows designers to design for high reliability, but it also permits them to quantitatively demonstrate reliability. According to Downer (2009), in engineering literature redundancy could be used as an indicator for reliability because it offers 'a powerful and convincing rubric' with which engineers could mathematically establish reliability levels much higher than they could derive from lab testing. Furthermore, Javanbarg and Takada (2007) highlighted the importance in assessing the redundancy of water networks from three perspectives. Firstly, it is very important to consider the redundancy in the network design stage to obtain the optimum network layout. Secondly, the insufficiency of redundancy could have a significant impact on the road transport network level of service, in addition to catastrophic consequences in the case of rapid evacuation (Immers et al. 2004). The third advantage according to Javanbarg and Takada (2007) is that the consideration of redundancy could help in finding the best recommended mitigation plans against different kind of disruptions.

Redundancy has a significant impact on the resilience of road transport networks as it represents the spare capacity of road transport networks under different scenarios (Lhomme et al. 2013). The link between redundancy and resilience concepts has been discussed in various disciplines. For example, Haimes (2009) suggested that a water distribution system could be resilient against a major storm that would shut down one of the power lines if it has redundancy in its electric power subsystem, whereas, Yazdani and Jeffrey (2012) considered redundancy along with the connectivity as the topological aspects of water network resilience. In computer science, Randles et al. (2011) reported that

Corresponding author: Rawia Ahmed El-Rashidy

E-mail: r.elrashidy@hud.ac.uk 
distributed redundancy improves complex system resilience and Anderson et al. (2011) suggested that the redundancy of road transport network is one of the resilience indicators.

The main aim of this paper is to propose a redundancy index that is able to account for the topology characteristics of road transport networks and the dynamic nature of traffic flow, while maintaining the advantages of easy implementation. The entropy concept that has been used in various disciplines to model redundancy has been employed for the first time, to develop road transport network redundancy indices. The paper initially presents a general review of the interpretation of redundancy in different disciplines. The development of the proposed redundancy index is then described along with a discussion of the entropy concept and its use in transport applications. Two case studies are given in order to investigate the implementation of the proposed redundancy index and to test its variations under different scenarios. The methodology also explores the need to develop an aggregated redundancy index in order to evaluate the redundancy of the overall network under different conditions.

\section{Survey of Redundancy Measures}

The concept of redundancy is well established in technological fields such as engineering, computer science, and system design (Streeter 1992). According to Streeter (1992), the redundancy characteristic of a system refers to its ability to self-organize, e.g. a process whereby internal structure and functions re-adjust along with changing circumstances. In engineering systems however, the redundancy of a system could be defined as the extent of degradation the system can suffer without losing some specified elements of its functionality (Kanno, Ben-Haim 2011). Meanwhile, in the transport context it is defined as the availability of several paths for each set of Origin Destination (OD) pairs in the road transport network. Moreover, Immers et al. (2004) used the redundancy concept to refer to the degree of spare capacity in the network. Meanwhile, Javanbarg and Takada (2007) suggested that the redundancy of the water distribution system does not only imply the availability of several paths but also includes the excess capacity, known in the literature as the spare capacity of the network. Furthermore, (Snelder et al. 2012) suggested two types of redundancy: active and passive redundancy. According to Snelder et al. (2012), alternative routes could be considered as 'active redundancy' that could be preserved under regular conditions by various measures such as road pricing or speed adjustments. For example, the M42 Active Traffic Management (ATM) project increases the capacity and reduces the variability of journey times by allowing the use of the hard shoulder between J3a and J7 together with Variable Mandatory Speed Limits (VMSL) during periods of peak demand (Sultan et al. 2008). Passive redundancy could be used to represent back-up options that are only used in case of disruptions. As a specific example, the use of fast train services, ferries, coaches to travel across Europe as a result of airline disruptions during the 2010 Eyjafjallajokull Volcano (eTN 2010). Furthermore, Immers et al. (2004) explained that redundancy could be a multi-level concept as follows:

- strategic level: coordination between activity patterns such as avoiding major road works during peak period or organized events;

- tactical level: coordination amongst multimodal transport services and networks, similar to passive redundancy explained above; this is also known as 'distributed redundancy' where different systems could deliver the same outcomes (Randles et al. 2011);

- operational level: to manage the supply-demand relationships in the road transport network by applying different intelligent transport systems (ITS); for example using variable message signs to advise travellers on alternative routes in the case of link closure due to an accident.

Despite the importance of redundancy at both strategic and tactical levels, the current research focuses on proposing an indicator to quantify the operational redundancy of the road transport network (i.e. active redundancy) that could feed into both levels. It has been noted that there is a lack of research into the redundancy concept in the case of road transport networks compared with other networks, such as water distribution networks and power networks. For example, there are several indices (Yazdani, Jeffrey 2012; Javanbarg, Takada 2007; Awumah et al. 1991; Hoshiya et al. 2004) that have been developed to investigate the redundancy in the water distribution network using the entropy concept.

In the road transport network, the redundancy concept could be evaluated by considering the static conditions of the network such as road density. Jenelius (2009) pointed out that a higher road density to some extent guarantees a higher availability of alternative paths. However, road density only reflects the impact of the supply side without considering the effect of changes in demand and traffic conditions. Furthermore, road density only considers the fully operational link status e.g. by adding the link length to the whole network length or subtracting link length when the link is fully closed. Hyder Consulting (2010) estimated the redundancy value of a link as the total number of motorways, $A$ roads, and $B$ roads within a 10 kilometre radius of the link (A roads - 'major roads intended to provide large-scale transport links within or between areas; B roads - roads intended to connect different areas, and to feed traffic between A roads and smaller roads on the network' (DfT 2011)). However, both approaches (i.e. Hyder Consulting 2010; Jenelius 2009) introduced static, purely topological indicators. They do not indicate the impact of different traffic conditions (e.g. the road density or the number of adjacent routes despite the traffic flow conditions of the alternatives) in estimating the redundancy of the link.

Graph theory has also been used to quantify the redundancy of networks by using a number of indices, 
such as a clustering coefficient and the number of independent routes (Boccaletti et al. 2006). The clustering coefficient, also known as transitivity, is a measure of redundancy as it represents the overall probability for the network to have interconnected adjacent nodes (Rodrigue et al. 2013), which could be measured by different indicators (Boccaletti et al. 2006). The clustering coefficient is a significant characteristic of road transport network redundancy, however, it only considers the directly neighbouring nodes or links and neglects possible capacity limitations which may restrict redundancy (Erath et al. 2009). Similarly, the number of independent routes is not an ideal measure of network redundancy as it is purely a topological measure and is based on an arbitrary threshold (Corson 2010).

Jenelius (2010) introduced a 'redundancy importance' concept as a new way to study the role of the link in network redundancy. The author quantified the importance of redundancy in two ways. Firstly, the importance of flow-based redundancy was calculated as the weighted sum of the difference in flow arising from the closure of all links in the network. Secondly, an impact based redundancy importance measure was computed as the weighted sum of the difference in the impact measure arising from the closure of all links in the network.

The above discussion highlights the lack of redundancy research in the transport context compared with the case for water distribution networks and power grids. Furthermore, the redundancy index developed should be able to account for the topological characteristics of road transport networks as well as the dynamic nature of traffic flow.

\section{A Redundancy Model}

Based on the previous discussion, the quantification of redundancy requires both traffic flow variations and network topology to be taken into account. In this research, the level of redundancy has been investigated at the 'node to node' level rather than at 'zone to zone'. By doing so, it is possible to identify critical nodes within the network that have low redundancy indices and their impact on the overall network redundancy. The proposed model of redundancy can, then, assist policy makers to evaluate the effectiveness of particular policies or to assess the impact of the implementation of new technologies, for example the ATM scheme introduced at Junction 3A in M42 motorway (see the second case study below).

There are many uncertainties associated with road transport networks under different operational conditions. These include the uncertainties related to the supply side (such as link flow under different operational conditions) in addition to uncertain demand. To deal with these uncertainties, the concept of information entropy is adopted as one way of measuring uncertainty in the road transport network. In the following section a brief introduction to the entropy concept is given, followed by an outline of its use in modelling systems.

\subsection{The Entropy Concept}

The concept of entropy was initially proposed by Shannon $(1948 \mathrm{a}, 1948 \mathrm{~b})$ to investigate the performance of communication channels and measure the uncertainties. The generic form of the entropy is as follows:

$$
H(x)=\sum_{i=1}^{n} p_{i} \cdot \ln \left(\frac{1}{p_{i}}\right),
$$

where: $H(x)$ is an entropic measure of a system $x ; n$ is the total number of the system elements under consideration; $p_{i}$ represents a system parameter that could be used to identify a certain characteristic of element $i$.

According to Swanson et al. (1997), the entropy measure suggested by Shannon (1948a, 1948b) is a good measure to quantify the existing number of degrees of freedom of a system. In general, the relative link flow is used as a system parameter (Javanbarg, Takada 2007). For example, if a node $J$ has a number of adjacent links $l$, then $p_{i}$ could be the relative flow of link $i$, e.g. flow $f_{i}$ of link $i$ divided by the total flow of node J, i.e.:

$$
p_{i}=\frac{f_{i}}{\sum_{k=1}^{l} f_{k}} \text {. }
$$

According to Wilson (1970) there are two main streams in the use of the entropy concept; namely a measure of some property of a system and a model building tool to maximise the available information. For example, the entropy concept is used widely in water distribution networks (Hoshiya, Yamamoto 2002), power grids (Koç et al. 2013) and computer networks (Randles et al. 2011). In transport literature, the entropy concept is widely accepted as a subjective measure to develop a trip distribution model using entropy-maximising methods (Wilson 1970). For example, Sun et al. (2012) proposed an entropy-based optimization approach to estimate the demand for transfers between the transport modes available in an intermodal transport terminal. Miao et al. (2011) developed an assessment model of capacity reliability for road network from the perspective of route entropy. Allesina et al. (2010) introduced a new quantitative measurement of complexity for a supply network using eight indices based on the entropy concept.

\subsection{Junction Redundancy Index}

Eq. (1) above is used here to develop a proposed redundancy index for nodes in the road transport network. Two redundancy indices are developed for each node; an outflow redundancy index $R I 1_{\text {out }}$ and an inflow redundancy index $R I 1_{\text {in }}$. $R I 1_{\text {out }}$ is estimated based on the outbound links whereas $R I 1_{\text {in }}$ is calculated based on the inbound links of a node, as given in Eqs (2) and (3) respectively, below:

$$
\begin{aligned}
& R I 1_{\text {out }}(O)=\frac{\sum_{b=1}^{k} \frac{f_{b m}^{i}}{\sum_{z=1}^{k} f_{z m}^{i}} \cdot \ln \frac{\sum_{z=1}^{k} f_{z m}^{i}}{f_{b m}^{i}}}{\ln (k)} ;
\end{aligned}
$$




$$
R I 1_{\text {in }}(O)=\frac{\sum_{a=1}^{n} \frac{f_{a m}^{i}}{\sum_{z=1}^{n} f_{z m}^{i}} \cdot \ln \frac{\sum_{z=1}^{n} f_{z m}^{i}}{f_{a m}^{i}}}{\ln (n)},
$$

where: $f_{b m}^{i}$ is the outbound flow of link $b$ during time interval $i$ using a travel mode $m, k$ is the total number of outbound links attached to node $O ; f_{a m}^{i}$ is the inbound flow of link a during time interval $i$ using a travel mode $m$ and $n$ is the total number of inbound links attached to node $O$ (Fig. 1).

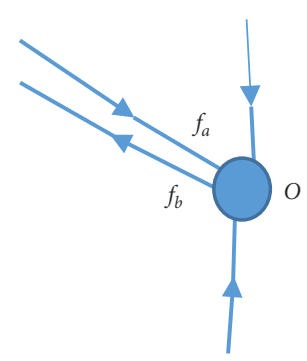

Fig. 1. Example illustrating the outbound and inbound flows of node $O$

The travel mode $m$ indicates different highway or public transport networks; however, in this research, the focus is on the highway network. The redundancy indices in Eqs (2) and (3) are normalized by $\ln (k)$ or $\ln (n)$ respectively, so as to have a range between 0 and 1 (Nagata, Yamamoto 2004; Corson 2010), provided that each link considered should have a traffic flow greater than $0\left(f_{b m}^{i}>0\right.$ and $\left.f_{a m}^{i}>0\right)$, i.e. links with zero traffic flow are not considered. The value of $R I 1_{\text {in }}$ or $R I 1_{\text {out }}$ is equal to 0 when either all traffic flow from or to node $o$ is assigned to one link, whilst the maximum value of node redundancy indicator is 1 , when the traffic flow is equally distributed over the attached links, as proved below.

Assuming a node $o$ has $k$ links where the inbound traffic flow of link $i$ is $f_{i}$ and the total inbound flow at the node is $F$, the inflow redundancy indicator $R I 1_{\text {in }}(O)$ using Eq. (3) is:

$$
\begin{aligned}
R I 1_{\text {in }}(O) & =\frac{\frac{f_{1}}{F} \cdot \ln \left(\frac{F}{f_{1}}\right)+\frac{f_{2}}{F} \cdot \ln \left(\frac{F}{f_{2}}\right)+\ldots+\frac{f_{n}}{F} \cdot \ln \left(\frac{F}{f_{n}}\right)}{\ln (n)} . \\
\text { As } 0 & <\frac{f_{i}}{F} \leq 1 \text {, therefore } R I 1_{\text {in }}(O) \geq 0 \text {. When } \frac{f_{i}}{F}=1 \text {, }
\end{aligned}
$$
other links are not assigned any traffic flow and $R I 1_{\text {in }}(O)=0$. Meanwhile, the maximum value of entropy is achieved when the flow over the attached links is equally distributed. In such a case, the inbound traffic flow of each link is:

$$
f_{1}=f_{2}=\ldots=f_{n}=\frac{F}{n} \text {. }
$$

Substituting the inbound traffic flow of each link in the above formula produces the inflow redundancy indicator $R I 1_{\text {in }}$ as follows:

$$
\begin{aligned}
& R I 1_{\text {in }}(O)=\frac{\frac{1}{n} \cdot \ln (n)+\frac{1}{n} \cdot \ln (n)+\ldots+\frac{1}{n} \cdot \ln (n)}{\ln (n)} ; \\
& R I 1_{\text {in }}(O)=\frac{n\left(\frac{1}{n} \ln (n)\right)}{\ln (n)} ; \\
& R I 1_{\text {in }}(O)=1 .
\end{aligned}
$$

The redundancy index $R I 1(O)$ of a node $o$ is eventually controlled by either $R I 1_{\text {in }}(O)$ or $R I 1_{\text {out }}(O)$. To identify the more influential redundancy index i.e. $R I 1_{\text {in }}(O)$ or $R I 1_{\text {out }}(O)$, the junction delay and junction volume capacity ratio are calculated for each direction (i.e. inbound and outbound) and correlated against the respective values of $R I 1_{\text {in }}(O)$ or $R I 1_{\text {out }}(O)$. The index most strongly correlated with these two junction levels of service identifies the junction redundancy level, as presented in section 4.1.1 below. The junction delay $J D_{\text {in }}^{i}(O)$, for inbound links is calculated by the following equation:

$$
J D_{i n}^{i}(O)=\frac{\sum_{a=1}^{n}\left(t_{a m}^{i}-T_{a m}^{i}\right) \cdot f_{a m}^{i}}{\sum_{z=1}^{k} f_{z m}^{i}},
$$

where: $t_{a m}^{i}$ is the actual travel time for inbound link $a$ during time interval $i$ using travel mode $m ; k$ is the total number of inbound links; $T_{a m}^{i}$ is the free flow travel time of inbound link $a$ during time interval $i$ using travel mode $m$. The junction volume capacity ratio $J V C R_{i n}^{i}(O)$, is calculated as:

$$
J \operatorname{VCR}_{i n}^{i}(O)=\frac{\frac{\sum_{a=1}^{n} f_{a m}^{i}}{\sum_{a=1}^{n} C_{a m}} \cdot f_{a m}^{i}}{\sum_{z=1}^{k} f_{z m}^{i}},
$$

where: $C_{a m}$ is the design capacity of link $a$ with mode $m$.

Similarly, the two Eqs (4) and (5) can also be adjusted to obtain junction delay and the volume capacity ratio for the outbound links.

\subsection{Analysis and Limitations of the Proposed Redundancy Index}

In this section, simple numerical examples are presented to examine the validity of the proposed $R I 1_{\text {in }}$ and $R I-$ $1_{\text {out }}$ in reflecting the topological properties of the node (e.g. number of attached links) in addition to traffic flow variation. Fig. 2a shows node $J$ with five links (2 inbound and 3 outbound links) whilst the traffic flow for each link is also shown in Fig. 2. Eqs (2) and (3) have been used to calculate $R I 1_{\text {out }}(J)$ and $R I 1_{\text {in }}(J)$ as 0.96 and 0.89 respectively, reflecting the impact of the increase in the number of outbound links. However, if the number of outbound and inbound links is the same 
but the flow distributions are different, e.g. node $O$ in Fig. $2 \mathrm{~b}, R I 1_{\text {in }}(O)$ increases to 0.94 due to the change in load distribution (i.e. change from $900 / 400$ to $830 / 470$ ), whereas $R I 1_{\text {out }}(O)$ significantly decreases to 0.78 due to the reduction of outbound links. This illustrates how the entropy concept reflects load distribution on the redundancy level. In general, the distribution of load between the adjacent links has a significant impact on the entropy value. A higher value of $H(x)$ presented in Eq. (1) could be obtained for the same total flow by the uniform distribution of the flow over the incident links, as concluded by Shannon (1948). For example, if the outbound flows of node $Z$ shown in Fig. $2 c$ are equally distributed over the two outbound links, $R I 1_{\text {out }}$ will be 1 , higher than a value for $R I 1_{\text {in }}$ of 0.90 in the case of a $580 / 270$ flow distribution. Doubling the flow on each link (with the same flow distribution between links) gives the same redundancy index. For example, $R I 1_{\text {in }}$ for node $Q$ (Fig. 2d) has the same value of 0.90 when the link flow increases to 1160 and 540 from 580 and 270 , as that shown for node $Z$ in Fig. 2 c.

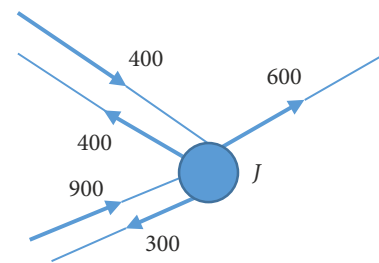

b)

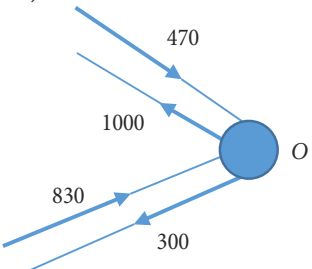

c)

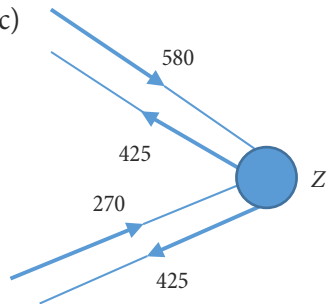

d)

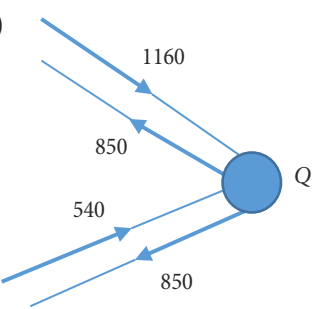

Fig. 2. Examples illustrating different traffic flow [veh/hr] and topology properties on redundancy index: a - node $J$; b - node $O$; c - node $Z$; d - node $Q$

This shortcoming of $R I 1_{\text {out }}$ and $R I 1_{\text {in }}$ (defined by Eqs (2) and (3)) highlights the need to introduce traffic flow variation compared with the link capacity in the definition of the redundancy index. In this respect, the redundancy index will then incorporate the link spare capacity in line with Immers et al. (2004). The next section introduces alternative redundancy indices to include the impact of link traffic conditions in the calculation of the redundancy of attached nodes.

\subsection{Impact of Link Spare Capacity and Travel Speed on Junction Redundancy}

To reflect the impact of increases/decreases in flow on node redundancy, the relative link spare capacity, $\rho_{a m}^{i}$ is introduced. For an inbound link $a, \rho_{a m}^{i}$ is represented by the percentage of the link spare capacity with respect to the node total spare capacity, as given by Eq. (6):

$$
\rho_{a m}^{i}=\frac{C_{a m}-f_{a m}^{i}}{\sum_{a=1}^{n}\left(C_{a m}-f_{a m}^{i}\right)} \cdot 100 .
$$

In addition to the impact of link spare capacity, link average travel speed should also be integrated to reflect the impact of the level of service on the redundancy index. As each link has its own free flow speed, the influence of link flow speed on junction redundancy is incorporated here using the Relative Link Speed (RLS) and calculated by the following equation:

$$
R L S(a)=\frac{v_{a m}}{V_{a m}},
$$

where: $v_{a m}$ is the average travel speed of link $a ; V_{a m}$ is the free flow travel speed of link $a$.

The redundancy indices proposed here are based on different logical combinations of relative link spare capacity, $\rho_{a m}^{i}$ and RLS. The main aim is to identify the best system parameters that can be used to develop a junction redundancy index, reflecting the junction topology and traffic flow conditions. Five additional redundancy indices are therefore introduced as given in Table 1. In $R I 2_{\text {in }}$ and $R I 6_{\text {in }}$ the relative link spare capacity $\rho_{a m}^{i}$ is used as the system parameter. However, in $R I 6_{i n}$, the calculated entropy for each link is weighted by the $R L S_{a}$, to account for the dynamic flow variation. In contrast the effect of the $R L S_{a}$, is included in the system parameter of $R I 3_{i n}$. The system parameter $p_{i}$ used in $R I 3_{\text {in }}$ is therefore given by the multiplication of the $R L S_{a}$ by the relative link spare capacity $\rho_{a m}^{i}$. The system parameter used in $R I 5_{\text {in }}$ is the $R L S_{a}$ multiplied by the relative link capacity with respect to the total junction capacity $C_{a m} / \sum_{a=1}^{n} C_{a m}$. In the final redundancy index $R I 4_{i n}$ considered, the relative link spare capacity $C_{a m}-f_{a m}^{i}$ to link capacity $C_{a m}$ has been employed as the system parameter. However the calculated entropy for each link has been weighted by the $R L S_{a}$ in a similar way to $R I \sigma_{i n}$.

Tables 2 and 3 show the flow of links and the values of $R I 1_{\text {in }}, R I 1_{\text {out }}, R I 2_{\text {in }}$ and $R I 2_{\text {out }}$ for the four nodes presented in Fig. 2 and two different road capacities of 1200 and $2200 \mathrm{veh} / \mathrm{hr}$, respectively. Other redundancy indices are not presented in Tables 2 and 3 as their calculation requires the value of RLS. The values of each link capacity $C_{a m}$, could vary based on the road type and speed limit. For example, $C_{a m}$ could be equal to 1200,1500 , or $1800 \mathrm{veh} / \mathrm{hr}$ in case of urban links whereas 2200 or 2400 $\mathrm{veh} / \mathrm{hr}$ is more appropriate for a motorway link type. In this numerical example, $C_{a m}$ is taken equal to 1200 $\mathrm{veh} / \mathrm{hr}$ (Table 2) and $2200 \mathrm{veh} / \mathrm{hr}$ (Table 3 ) to investigate the impact of link capacity on the redundancy indices. Taking the impact of spare capacity into account leads to a decrease in the redundancy index when the flow increases; however, its importance is highlighted when the flow doubles but has the same distribution (Table 2).

For example in Table 2, nodes $Z$ and $Q$ have the same number of links but double the flow, consequently 
$R I 2_{\text {in }}(Q)$ is decreased compared with $R I 2_{\text {in }}(Z)$, whereas $R I 2_{\text {in }}(Q)$ is equal to $R I 2_{\text {in }}(Z)$. Furthermore, the outbound flow for both nodes, $Z$ and $Q$ are equally distributed over the two outbound links, leading to the same $R I 1_{\text {out }}$ and $R I 2_{\text {out }}$ for the two nodes $Z$ and $Q$. This reflects the ability of $R I 2_{\text {in }}$ to consider the impact of flow increases, other than in the case of equally distributed flow. To investigate the impact of flow distribution on node redundancy, node $O$ has an inbound flow distribution different to that of the outbound flow. This leads to different inbound and outbound redundancy indices. It has been found that the increase in a link flow compared with the other adjacent links leads to a decrease in the redundancy indices even though the total flow remains the same. To investigate the impact of the number of links adjacent to the node, node $J$ has been introduced with 2 inbound links, meanwhile the number of out- bound links are 3 . Consequently both indices, $R I 1_{\text {out }}$ and $R I 2_{\text {out }}$ are higher than the inbound redundancy indices $R I 1_{\text {in }}$ and $R I 2_{\text {in }}$, respectively, reflecting the ability of both indices to represent the topological aspects of nodes.

Comparing Tables 2 and 3, the increase in link capacity (from 1200 to $2200 \mathrm{veh} / \mathrm{hr}$ ) leads to an increase in $R I 2_{\text {in }}$ and $R I 2_{\text {out }}$ of different percentages, whereas $R I 1_{\text {in }}$ and $R I 1_{\text {out }}$ are the same for each node. For example, $R I 2_{\text {in }}$ and $R I 2_{\text {out }}$ of nodes $J, O, Z$ and $Q$ increase due to capacity increases and as other properties such as flow distribution and total flow remain the same.

The suitability of the redundancy indices presented in Table 1 is further applied on two case studies, namely a synthetic road transport network of Delft city and Junction 3A of the M42 motorway near Birmingham, as explained in Section 4 of the paper.

Table 1. System parameters used in the six redundancy indices considered

\begin{tabular}{|c|c|c|c|}
\hline $\begin{array}{l}\text { Redun- } \\
\text { dancy } \\
\text { index }\end{array}$ & System parameter & Redundancy index formulation & System parameter explanation \\
\hline$R I 1_{\text {in }}$ & $p_{i}=\frac{f_{a m}^{i}}{\sum_{z=1}^{n} f_{z m}^{i}}$ & $R I 1_{i n}(O)=\frac{\sum_{a=1}^{n} \frac{f_{a m}^{i}}{\sum_{z=1}^{n} f_{z m}^{i}} \cdot \ln \left(\frac{\sum_{z=1} f_{z m}^{i}}{f_{a m}^{i}}\right.}{\ln (n)}$ & $\begin{array}{l}\text { Link flow } f_{a m}^{i} \text { with respect to the total } \\
\text { junction flow } \sum_{z=1}^{n} f_{z m}^{i}\end{array}$ \\
\hline$R I 2_{\text {in }}$ & $p_{i}=\rho_{a m}^{i}$ & $R I 2_{\text {in }}(O)=\frac{\sum_{a=1} \rho_{a m}^{i} \cdot \ln \left(\frac{1}{\rho_{a m}^{i}}\right)}{\ln (n)}$ & Relative link spare capacity $\rho_{a m}^{i}$ \\
\hline$R I 3_{\text {in }}$ & $p_{i}=R L S_{a} \cdot \rho_{a m}^{i}$ & $R I 3_{i n}(O)=\frac{\sum_{a=1}^{n}\left(R L S_{a} \cdot \rho_{a m}^{i}\right) \cdot \ln \left(\frac{1}{R L S_{a} \cdot \rho_{a m}^{i}}\right)}{\ln (n)}$ & $\begin{array}{l}R L S_{a} \text { multiplied by relative link spare } \\
\text { capacity } \rho_{a m}^{i}\end{array}$ \\
\hline$R I 4_{\text {in }}$ & $p_{i}=\frac{C_{a m}-f_{a m}^{i}}{C_{a m}}$ & $R I 4_{i n}(O)=\frac{\sum_{a=1}^{n} R L S_{a}\left(\frac{C_{a m}-f_{a m}^{i}}{C_{a m}}\right) \cdot \ln \left(\frac{C_{a m}}{C_{a m}-f_{a m}^{i}}\right)}{\ln (n)}$ & $\begin{array}{l}\text { Relative spare capacity } C_{a m}-f_{a m}^{i} \text { to link } \\
\text { capacity } C_{a m} \text {. However, the calculated en- } \\
\text { tropy for each link is weighted by the } R L S_{a}\end{array}$ \\
\hline$R I 5_{\text {in }}$ & $p_{i}=R L S_{a} \cdot \frac{C_{a m}}{\sum_{a=1}^{n} C_{a m}}$ & $R I 5_{i n}(O)=\frac{\sum_{a=1}^{n}\left(R L S_{a} \cdot \frac{C_{a m}}{\sum_{a=1}^{n} C_{a m}}\right) \cdot \ln \left(\frac{\sum_{a=1}^{n} C_{a m}}{R L S_{a} \cdot C_{a m}}\right.}{\ln (n)}$ & $\begin{array}{l}R L S_{a} \text { multiplied by relative link capacity } \\
\text { with respect to the total junction capacity } \\
\frac{C_{a m}}{\sum_{a=1}^{n} C_{a m}}\end{array}$ \\
\hline$R I 6_{\text {in }}$ & $p_{i}=\rho_{a m}^{i}$ & $R I 6_{i n}(O)=\frac{\sum_{a=1}^{n} R L S_{a}\left(\rho_{a m}^{i}\right) \cdot \ln \left(\frac{1}{\rho_{a m}^{i}}\right)}{\ln (n)}$ & $\begin{array}{l}\text { Relative link spare capacity } \rho_{a m}^{i} \cdot \\
\text { However, the calculated entropy for each } \\
\text { link is weighted by the } R L S_{a}\end{array}$ \\
\hline
\end{tabular}

Table 2. Redundancy indices for nodes shown in Fig. 2 using $c_{a m}=1200 \mathrm{veh} / \mathrm{hr}$

\begin{tabular}{|c|c|c|c|c|c|c|}
\hline Node & Inbound links flow & $R I 1_{\text {in }}$ & $R I 2_{\text {in }}$ & Outbound links flow & $R I 1_{\text {out }}$ & $R I 2_{\text {out }}$ \\
\hline$J$ & $900 / 400$ & 0.89 & 0.85 & $600 / 400 / 300$ & 0.96 & 0.99 \\
\hline$O$ & $830 / 470$ & 0.94 & 0.92 & $1000 / 300$ & 0.78 & 0.68 \\
\hline$Z$ & $580 / 270$ & 0.90 & 0.97 & $425 / 425$ & 1.00 & 1.00 \\
\hline$Q$ & $1160 / 540$ & 0.90 & 0.32 & $850 / 850$ & 1.00 & 1.00 \\
\hline
\end{tabular}


Table 3. Redundancy indices for nodes shown in Fig. 2 using $c_{a m}=2200 \mathrm{veh} / \mathrm{hr}$

\begin{tabular}{|c|c|c|c|c|c|c|}
\hline Node & Inbound links flow & $R I 1_{\text {in }}$ & $R I 2_{\text {in }}$ & Outbound links flow & $R I 1_{\text {out }}$ & $R I 2_{\text {out }}$ \\
\hline$J$ & $900 / 400$ & 0.89 & 0.98 & $600 / 400 / 300$ & 0.96 & 1.00 \\
\hline$O$ & $830 / 470$ & 0.94 & 0.99 & $1000 / 300$ & 0.78 & 0.96 \\
\hline$Z$ & $580 / 270$ & 0.90 & 0.99 & $425 / 425$ & 1.00 & 1.00 \\
\hline$Q$ & $1160 / 540$ & 0.90 & 0.96 & $850 / 850$ & 1.00 & 1.00 \\
\hline
\end{tabular}

\section{Network Redundancy Index}

Despite the importance of the node redundancy based index in identifying nodes with low redundancy, there is still a need, however, for an aggregated redundancy index in order to evaluate the redundancy of the whole network under different conditions. A network redundancy indicator could be used to assess the effectiveness of different policies or technologies on the improvement of overall network redundancy. Furthermore, an evaluation of the network redundancy using a single index can help in comparing network redundancy level under different conditions, as explained in case study 1 below.

The redundancy indices, $R I 1_{\text {in }}(O)$ and $R I 1_{\text {out }}(O)$, for all the nodes in the road transport network are calculated first. A network redundancy index $N R I_{\text {in }}$ is developed by summing a weighted $R I s_{i n}$ for all the nodes in the network as given in Eqs (8) and (9) below. The weight considered in the equations below is the node flow with respect to the total network flow:

$$
\begin{aligned}
& N R I_{\text {in }}=\sum_{o=1}^{N} \frac{f_{o m}^{i}}{\sum_{o=1}^{N} f_{\text {om }}^{i}} \cdot R I s_{\text {in }}(O) ; \\
& N R I_{\text {out }}=\sum_{o=1}^{N} \frac{f_{\text {om }}^{i}}{\sum_{o=1}^{N} f_{\text {om }}^{i}} \cdot R I s_{\text {out }}(O),
\end{aligned}
$$

where: $f_{o m}^{i}$ is the total flow of node $o$ during the time interval $i$ using a travel mode $m$; $N$ is the total number of nodes in the road transport network.

\section{Application Case Studies}

\subsection{Case Study 1: Delft Road Transport Network}

A synthetic road transport network of Delft city is used to illustrate the redundancy of road network under different scenarios using the proposed methodology. The Delft road transport network consists of 25 zones, two of which are under development (24 and 25) and 1142 links. 483 links are bi-directional and 176 are one-way including connectors and different road types as depicted in Fig. 3. The Delft road transport network demonstrates a realistic network size, in addition to the availability of socioeconomic data of Delft in OmniTrans modelling software (version number 6.024). A full description of the Delft city road transport network is given in ElRashidy and Grant-Muller (2014), which was concerned with measuring the vulnerability of the network.

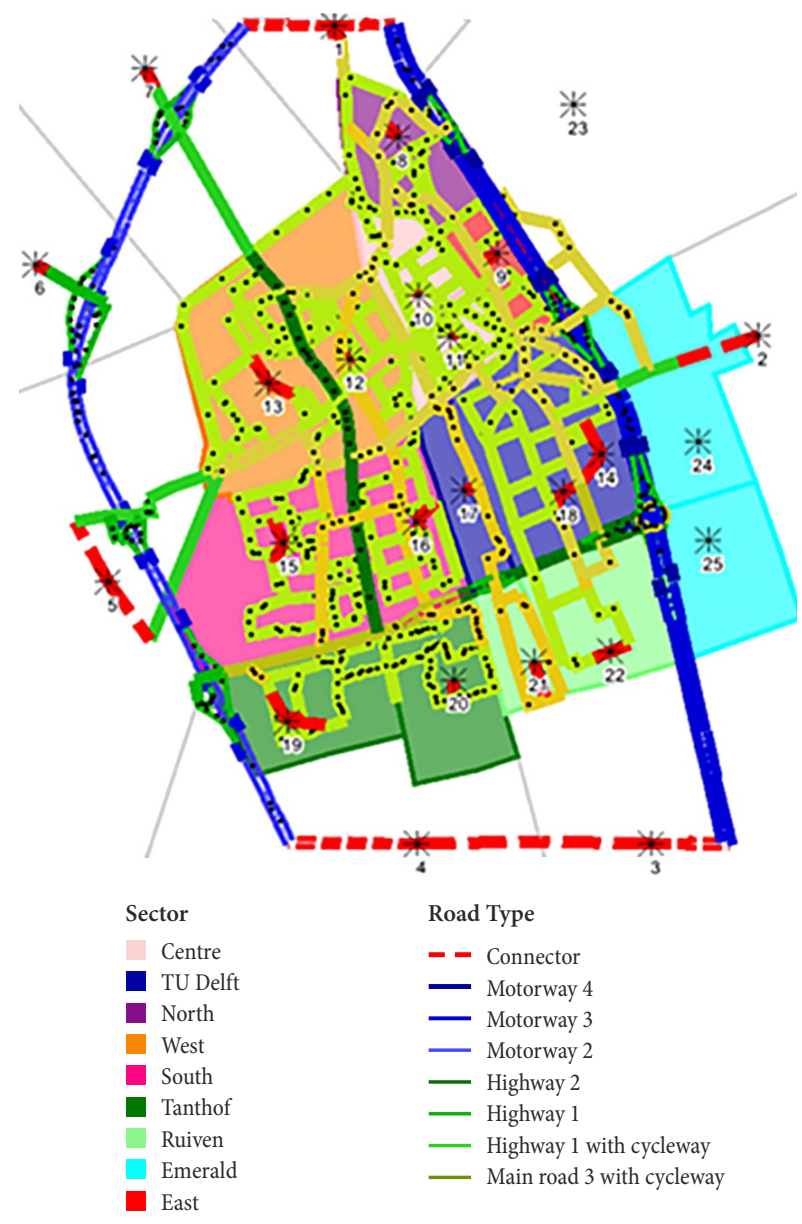

Fig. 3. The synthetic road transport network of Delft city

\subsubsection{Redundancy Indices of Various Nodes in Delft Road Network}

In the case study undertaken here the OmniTrans modelling software (version number 6.024) has been employed to obtain the spatial distribution of the traffic volume using the User Equilibrium (UE) assignment. UE is based on Wardrop's first principle whereby no individual trip maker can reduce his/her path cost by switching routes. This principle is also known as the user optimum (Wardrop 1952). The mathematical formulation of UE is explained in detail by Ortúzar and Willumsen (2011). Junction modelling available in $\mathrm{Om}$ niTrans modelling software is also integrated with UE model to enhance the network simulation.

The output from OmniTrans modelling software (version number 6.024) includes traffic flow in vari- 
ous links connected to each network node. A computer programme has been developed using MatLab R2011a (http://se.mathworks.com) to calculate $R I_{\text {out }}$ and $R I_{\text {in }}$ for each node using the different equations presented in Table 1.

The proposed indices are calculated under the same network and traffic conditions to test the ability of the index to reflect the redundancy concept. The aim of using different performance parameters is to find out the most suitable one to develop the redundancy index. Each proposed index is calculated for each junction using MatLab R2011a code and compared with the junction delay in adjacent links. For example, the inbound redundancy index of a junction is compared with the junction delay for inbound links, whereas the outbound redundancy index of this node is compared with the junction delay of outbound links. Furthermore, in the case of a strong correlation between a redundancy index and junction delay or volume capacity ratio, each redundancy index is classified according to the junction type and investigated further. The following analysis focuses on $R I_{\text {in }}$ only, given that there was no correlation between any $R I_{\text {out }}$ and either the junction delay or volume capacity ratio.

Table 4 lists the correlation coefficient $r$ between the proposed redundancy indices and either the junction delay or volume capacity ratio. $r$ is a statistical measure of the degree to which two variables are linearly related. Table 4 indicates a strong correlation between the redundancy indices $R I 2_{i n}, R I 3_{i n}$ and $R I 6_{i n}$ and both the junction delay and volume capacity ratio. In contrast, $R I 1_{\text {in }}$ and $R I 5_{\text {in }}$ exhibit a very low correlation with both the junction delay and volume capacity ratio. Furthermore, $R I 4_{\text {in }}$ is strongly positively correlated with the junction volume capacity ratio $(r=0.95)$, indicating the unsuitability of $R I 4_{\text {in }}$ to model junction redundancy, as redundancy should be inversely proportional to the junction volume capacity. $R I 6_{i n}, R I 3_{\text {in }}$ and $R I 2_{\text {in }}$ exhibit moderate correlation with the junction volume capacity ratio $(-0.76,-0.71$ and -0.69 , respectively). The above analysis led to the exclusion of $R I 1_{i n}, R I 4_{i n}$ and $R I 5_{i n}$ as redundancy indices from any further analysis.

Table 5 gives a summary of $r$ values of the remaining three redundancy indices for different junction types. In general it suggests that $R I 3_{\text {in }}$ and $R I 6_{\text {in }}$ are the most suitable redundancy indices as they can reflect junction delay and volume capacity ratio for different junction types, as indicated by the high value of $r$. Furthermore, the analysis of $R I 2_{\text {in }}$ based on junction type shows that there is variation from one junction type to another.
Table 4. Correlation coefficient $r$ of various redundancy indices with junction delay $J D$ and volume capacity ratio $v / c$

\begin{tabular}{|c|c|c|}
\hline Redundancy index & $J D$ & $v / c$ \\
\hline$R I 1_{\text {in }}$ & 0.00 & 0.42 \\
\hline$R I 2_{\text {in }}$ & -0.71 & -0.69 \\
\hline$R I 3_{\text {in }}$ & -0.77 & -0.71 \\
\hline$R I 4_{\text {in }}$ & 0.35 & 0.95 \\
\hline$R I 5_{\text {in }}$ & -0.25 & -0.40 \\
\hline$R I 6_{\text {in }}$ & -0.77 & -0.76 \\
\hline
\end{tabular}

Note: +ive and -ive correlation coefficients indicate that, as $J D$ or $v / c$ increases, $R I$ increases and decreases, respectively.

For example, the highest $r, 0.87$, between $R I 2_{\text {in }}$ and total junction delay is for an equal priority junction type and roundabout junction type (Table 5 ). The lowest value of $r=0.49$ between $R I 2_{\text {in }}$ and total junction delay is for a give-way junction type, as depicted in Table 5. Similarly, the correlation between $R I 2_{\text {in }}$ and junction volume capacity ratio varies according to the junction type.

$r$ for $R I 3_{\text {in }}$ with junction delay for all junction types is higher than those for $R I 2_{i n}$, except for the roundabout junction type (which decreases by $2.4 \%$ ). The highest increase occurs for the give-way junction type, where $r$ increases by $67 \%$ (Table 5). Regarding the correlation between $R I 3_{\text {in }}$ and junction volume capacity ratio, two junction types (i.e. equal priority and give-way junction types), show some improvement over $R I 2_{\text {in }}$ (Table 5). For the other two types (i.e. signalized junction and roundabout), the $r$ value between $R I 3_{\text {in }}$ and the junction volume capacity ratio has declined compared to that between $R I 2_{\text {in }}$ and junction volume capacity ratio. Table 5 also confirms the high correlation of $R I 6_{\text {in }}$ with junction delay and junction volume capacity ratio for different junction types. Overall, Table 5 indicates that the suitability of each redundancy index relies on the junction type. However, $R I 2_{\text {in }}$ has generally a lower correlation with junction delay and the junction volume capacity ratio for different junction types than either $R I 3_{i n}$ or $R I 6_{i n}$. As a result, $R I 3_{\text {in }}$ and $R I 6_{\text {in }}$ are examined further below.

In the following, both $R I 3_{\text {in }}$ and $R I 6_{\text {in }}$ are calculated for a small number of junctions from the synthetic Delft road network to show their validity. $R I 3_{\text {in }}$ and $R I 6_{\text {in }}$ have been selected as they exhibited a reasonably consistent performance for various junction types. Table 6 shows four selected junctions from the synthetic Delft road network with the flow, average speed, free flow speed and capacity of their inbound links along with the calculated values of $R I 3_{\text {in }}$ and $R I 6_{i n}$. The calculated values

Table 5. Summary of $r$ of various redundancy indices with junction delay $J D$ and volume capacity ratio $v / c$

\begin{tabular}{|c|c|c|c|c|c|c|c|c|}
\hline \multirow{2}{*}{ Redundancy index } & \multicolumn{9}{|c|}{ Junction type } \\
\cline { 2 - 10 } & \multicolumn{2}{|c|}{ Equal priority } & Give way junction & \multicolumn{2}{c|}{ Signalized junction } & \multicolumn{2}{c|}{ Roundabout junction } \\
\cline { 2 - 10 } & JD & $v / c$ & JD & $v / c$ & JD & $v / c$ & JD & $v / c$ \\
\hline$R I 2_{\text {in }}$ & 0.87 & 0.66 & 0.49 & 0.50 & 0.69 & 0.85 & 0.87 & 0.90 \\
\hline$R I 3_{\text {in }}$ & 0.89 & 0.77 & 0.82 & 0.70 & 0.70 & 0.63 & 0.85 & 0.72 \\
\hline$R I 6_{\text {in }}$ & 0.90 & 0.77 & 0.81 & 0.78 & 0.71 & 0.71 & 0.85 & 0.63 \\
\hline
\end{tabular}


Table 6. $R I 3_{\text {in }}$ and $R I 6_{\text {in }}$ values for selected nodes in road transport network of Delft city

\begin{tabular}{|c|c|c|c|c|c|c|c|c|}
\hline \multirow{2}{*}{$\begin{array}{c}\text { Node } \\
\text { number }\end{array}$} & \multicolumn{4}{|c|}{ Inbound links } & \multirow{2}{*}{$\begin{array}{c}\text { Junction } \\
\text { delay } \\
\text { [veh/min] }\end{array}$} & \multirow{2}{*}{\begin{tabular}{|c|} 
Junction \\
volume capacity \\
ratio
\end{tabular}} & \multirow[b]{2}{*}{$R I 3_{\text {in }}$} & \multirow[b]{2}{*}{$R I 6_{\text {in }}$} \\
\hline & $\begin{array}{c}\text { Link flow } \\
\text { [veh/hr] }\end{array}$ & $\begin{array}{c}\text { Link capacity } \\
\text { [veh/hr] }\end{array}$ & $\begin{array}{c}\text { Link speed } \\
{[\mathrm{km} / \mathrm{hr}]}\end{array}$ & $\begin{array}{l}\text { Link free flow } \\
\text { speed }[\mathrm{km} / \mathrm{hr}]\end{array}$ & & & & \\
\hline \multirow{2}{*}{5001} & 198 & 1800 & 50 & 50 & \multirow{2}{*}{0} & \multirow{2}{*}{0.07} & \multirow{2}{*}{1} & \multirow{2}{*}{1} \\
\hline & 41.04 & 1800 & 50 & 50 & & & & \\
\hline \multirow{3}{*}{6856} & 773 & 1200 & 29.86 & 35 & \multirow{3}{*}{23.53} & \multirow{3}{*}{0.26} & \multirow{3}{*}{0.91} & \multirow{3}{*}{0.88} \\
\hline & 142 & 1200 & 35 & 35 & & & & \\
\hline & 32 & 1200 & 35 & 35 & & & & \\
\hline \multirow{3}{*}{6983} & 293 & 2200 & 70 & 70 & \multirow{3}{*}{219.33} & \multirow{3}{*}{0.56} & \multirow{3}{*}{0.75} & \multirow{3}{*}{0.67} \\
\hline & 1844 & 2200 & 55.4 & 70 & & & & \\
\hline & 1538 & 2200 & 61.8 & 70 & & & & \\
\hline \multirow{3}{*}{7094} & 1483 & 1800 & 35.7 & 50 & \multirow{3}{*}{341.72} & \multirow{3}{*}{0.35} & \multirow{3}{*}{0.81} & \multirow{3}{*}{0.79} \\
\hline & 225 & 1500 & 39.98 & 40 & & & & \\
\hline & 88 & 2800 & 50 & 50 & & & & \\
\hline
\end{tabular}

of both redundancy indices show the impact of spare capacity and speed variations. For example, node 5001 is connected with two inbound links with a very low traffic flow compared with their link capacity (i.e. junction volume capacity ratio $=0.07$ ) and average speed equal to free flow speed (junction delay $=0 \mathrm{veh} / \mathrm{min}$ ) exhibits a maximum value of $R I 3_{\text {in }}=1$ and $R I 6_{\text {in }}=1$. Node 6856 has 3 inbound links with a slightly high traffic flow compared with link capacity $(=0.64)$ in one link, causing a reduction in its average speed (junction delay $=$ $23.53 \mathrm{veh} / \mathrm{min}$ and junction volume capacity ratio $=$ 0.26 ), and therefore, $R I 3_{i n}=0.91$ and $R I 6_{\text {in }}=0.88$. Furthermore, node 6983 connected with inbound links has a higher junction delay time and volume capacity ratio than node 6856 , consequently, its $R I 3_{\text {in }}$ and $R I 6_{\text {in }}$ are lower than node 6858 redundancy indices as presented in Table 6. Furthermore, to compare the effect of the variation in junction delay and the volume capacity ratio on the redundancy indices, node 7094 was chosen as it has a higher junction delay and lower volume capacity ratio than node 6983 . The calculated values of $R I 3_{\text {in }}$ and $R I 6_{\text {in }}$ for junction 7094 are 0.81 and 0.79 respectively. These are higher than the calculated redundancy indices for junction 6983, indicating that both indices experienced more sensitivity to the increase in junction volume capacity ratio than the increase in junction delay.

\subsubsection{Impact of Demand Variations on Redundancy Indices of Delft Road Network}

The impact of variations in demand on $R I 3_{\text {in }}$ and $R I 6_{\text {in }}$ in addition to the network redundancy index for the Delft road transport network was investigated using different departure rates during the morning peak. $R I 3_{\text {in }}$ and $R I \sigma_{\text {in }}$ were calculated from the equations presented in Table 1, whereas Eq. (8) is implemented to calculate the network redundancy indices $N R I 3_{\text {in }}$ and $N R I 6_{i n}$.

Fig. 4 shows the variations of $N R I 3_{\text {in }}$ and NRI ${ }_{\text {in }}$ under uniformly distributed departure rates, whilst Fig. 5 plots the variations of $N R I 3_{\text {in }}$ and $N R I 6_{\text {in }}$ under different departure rates. Fig. 4 shows that as the load rate stays constant, $N R I 3_{\text {in }}$ and $N R I 6_{\text {in }}$ are also constant; however, $N R I 3_{\text {in }}$ is larger than $N R I 6_{i n}$. Otherwise, the redundancy level measured by $N R I 3_{\text {in }}$ and $N R I 6_{\text {in }}$ follows an opposite trend to the departure rate as depicted in Fig. 5, i.e. decreases with the departure rate increase. Similarly, both network indices, $\mathrm{NRI}_{\text {in }}$ and $\mathrm{NRI}_{\text {in }}$ follow an opposite trend to the total delay [veh/hr] as shown in Fig. 6. This leads to the conclusion that the proposed network indices $N R I 3_{\text {in }}$ and $N R I 6_{\text {in }}$ are able to reflect the impact of demand variation under the same network condition.

\subsubsection{Impact of Supply Variations on Redundancy Indices of Delft Road Network}

In this analysis the ability of NRI $3_{\text {in }}$ and $N R I 6_{\text {in }}$ to capture the impact of reductions in network capacity under the same variations of demand is examined. Overall network capacity could be reduced in real life conditions due to the effect of network wide events such as heavy rain or snowfall. This group of scenarios was undertaken using a reduced capacity of 2, 4 and $10 \%$ in order to model the impact of a weather related event. Fig. 7 shows the variations in the network redundancy index NRI3, for the variations in supply (as stated above) and the same variation in departure rate shown in Fig. 5. NRI3 shows variations during the modelling period (7:00 am - 9:00 am) in the case of reduced capacity compared with full network capacity as depicted in Fig. 7. In general, the largest reduction of network redundancy level occurs at $10 \%$ capacity reduction (see the difference between $N R I 3_{\text {in }}$ calculated for full capacity and $N R I 3_{\text {in }}$ for $10 \%$ capacity reduction) under different departure rates. Fig. 8 presents the total delay for the full network condition in addition to the reduced capacity scenarios. Fig. 7 and 8 indicate that the network redundancy for different network conditions follows an opposite trend as the total delay for the same network conditions. For example at 7:30 am, NRI $3_{\text {in }}$ and the total delay for the network at: (a) full capacity, (b) $2 \%$ and (c) $4 \%$ reduction are almost the same. When the network capacity reduction increased to (d) $10 \%$, more delay is experienced by the network and $N R I 3_{\text {in }}$ is lower than the previous cases. 


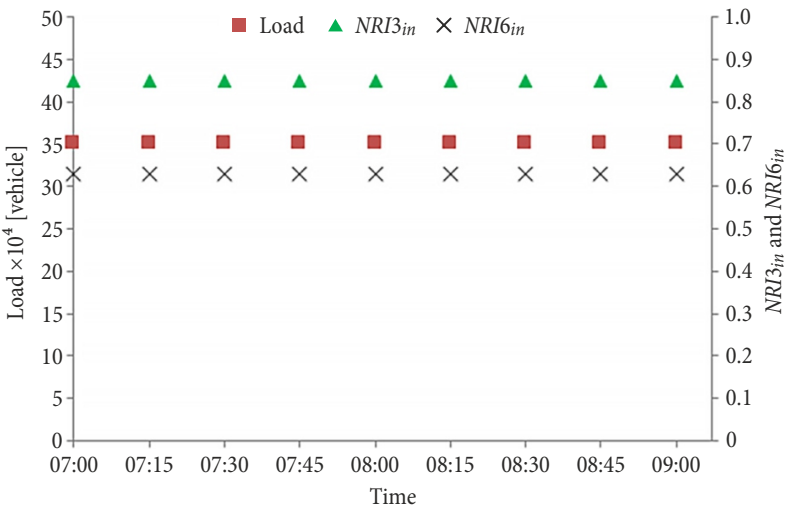

Fig. 4. $N R I 3_{\text {in }}$ and $N R I 6_{\text {in }}$ under uniform distributed departure rates

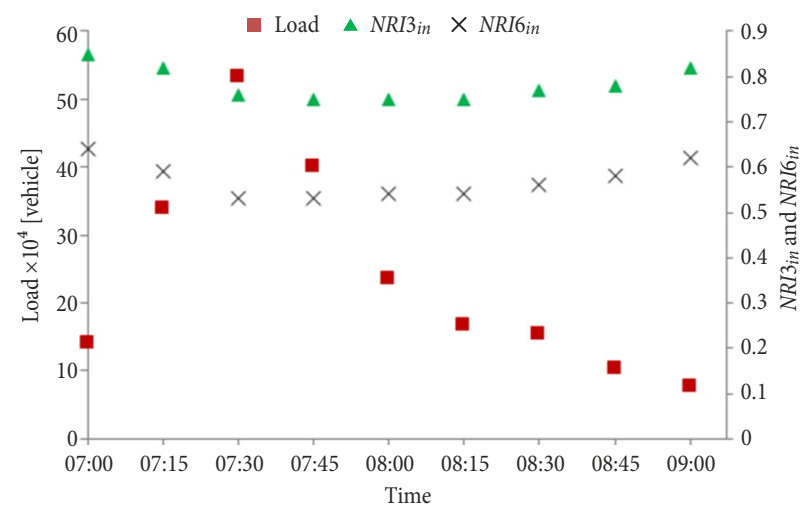

Fig. 5. NRIs and network load under different departure rates

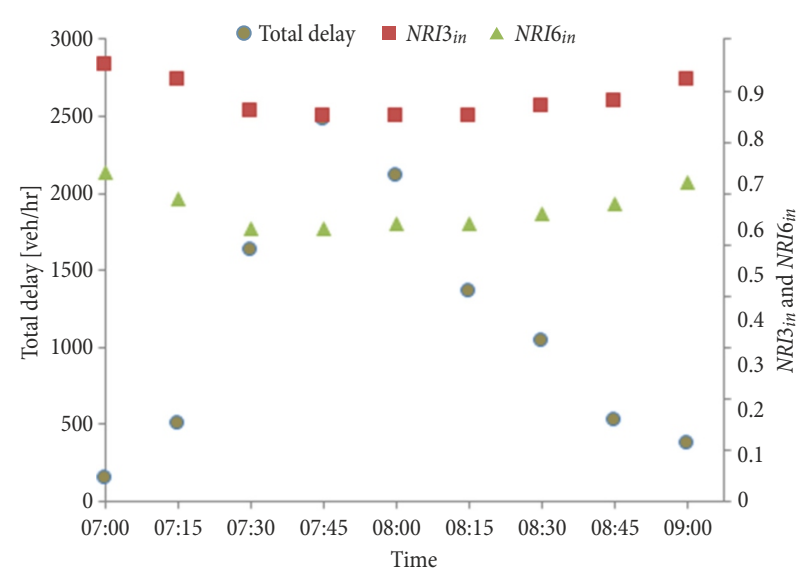

Fig. 6. $N R I 3_{\text {in }}$ and $N R I 6_{\text {in }}$ and total delay under different departure rates

\subsection{Case Study 2: Junction 3A in M42}

Junction 3a in M42 motorway shown in Fig. 9 was also employed to investigate the applicability of the proposed redundancy indices to reflect real life conditions. The choice of Junction 3a in M42 is due to the fact that the junction was a part of ATM scheme by the Highways Agency in 2006, therefore it is possible to study the variation of redundancy under different conditions. The scheme has enhanced the performance of M42 between

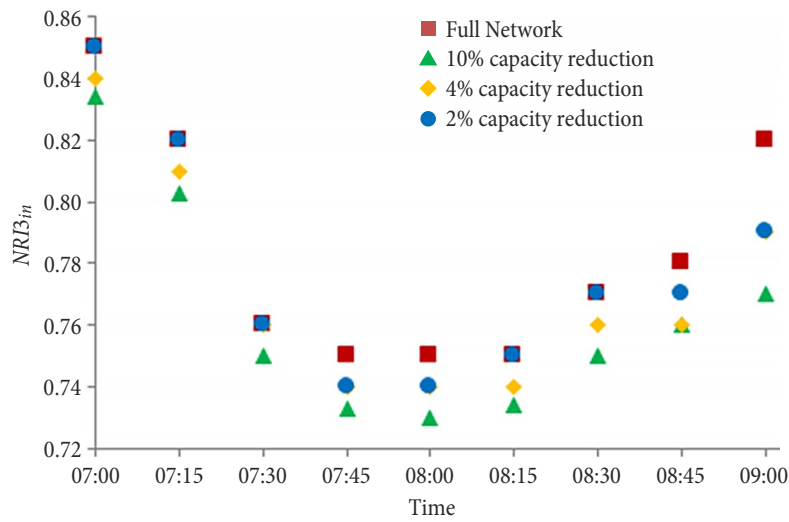

Fig. 7. NRI under different departure rates and network capacity

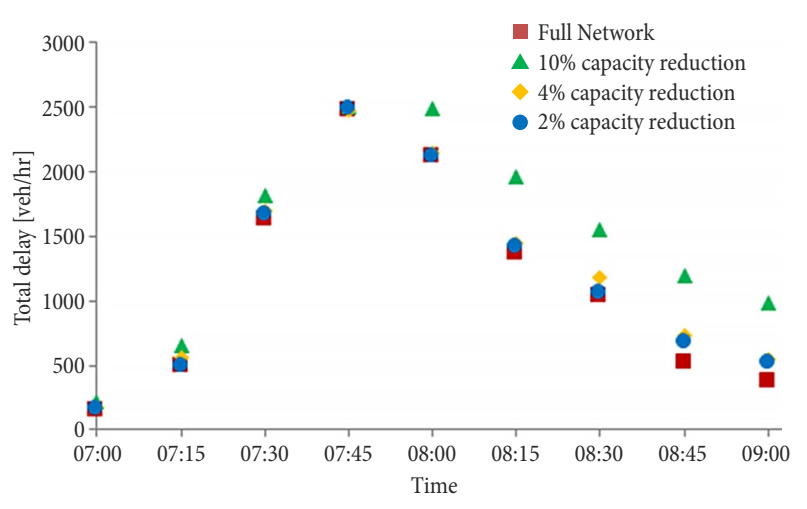

Fig. 8. Total delay under different capacity reduction

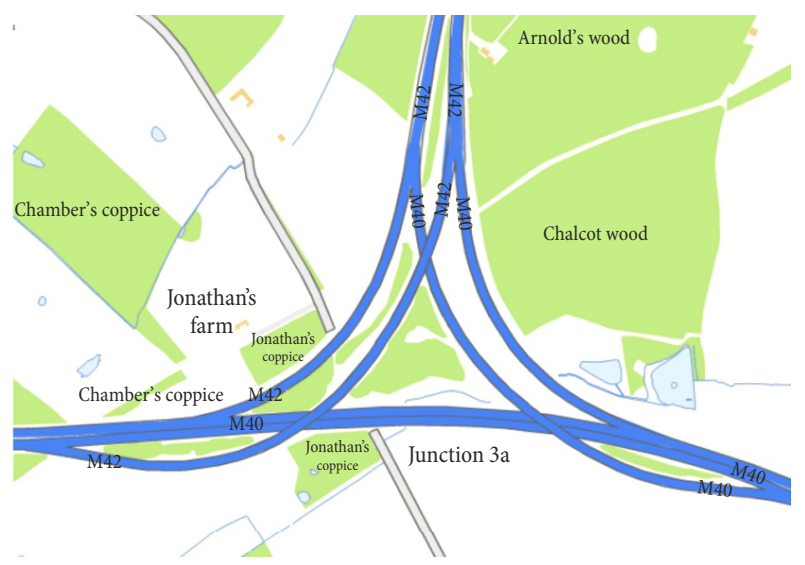

Fig. 9. Junction 3a in M42 motorway near Birmingham (C) Crown Copyright and database rights 2014; an Ordnance Survey/EDINA-supplied service)

J3a and $\mathrm{J} 7$ by the temporary usage of the hard shoulder to increase the route capacity from 3 lanes $(3 \mathrm{~L})$ to 4 lanes (4L), jointly with the use of VMSL during periods of peak demand (Mot 2008). In this study, four time periods were chosen to check the scheme effectiveness i.e. from October 2002 to April 2003 (NO-VMSL), from January 2006 to April 2006 (3L-VMSL), from October 2006 to April 2007 (4L-VMSL), and from January 2007 to April 2007 (4L-VMSL), as indicated in Table 7. 
Table 7. Time periods considered for scheme effectiveness

\begin{tabular}{|l|l|}
\hline \multicolumn{1}{|c|}{ Comparison Task } & \multicolumn{1}{c|}{ Time period } \\
\hline $\begin{array}{l}\text { NO-VSML against } \\
\text { 4L-VMSL }\end{array}$ & October 2002 to April 2003 \\
\hline October 2006 to April 2007 \\
\hline 4L-VMSL against & January 2006 to April 2006 \\
\hline
\end{tabular}

According to Sultan et al. (2008), the period October 2006 to April 2007 could be a suitable period to represent the influence of the full scheme, 4 lanes jointly with VMSL (4L-VMSL). Furthermore, the period October 2002 to April 2003 represent the pre-scheme period (NO-VMSL). Furthermore, the periods January 2006 to April 2006 and January 2007 to April 2007 could be implemented to compare between $3 \mathrm{~L}-\mathrm{VMSL}$ and $4 \mathrm{~L}-$ VMSL, respectively.

\section{Redundancy Index of Junction $3 A$ in M42}

The traffic flow parameters (i.e. link flow, speed, capacity and free flow speed), on the attached links of J3a were used to calculate $R I 3_{\text {in }}$ and junction delay. Data for the analysis had been collected from the Journey Time Database (JTDB) which is part of the Highways Agency Traffic Information System (HATRIS) (Highways Agency 2013).

The database included journey time, speed and traffic count data for the motorway and all-purpose trunk road network in England. Data were provided at $15 \mathrm{~min}$ intervals. For each time period, Sundays and Saturdays were excluded from the analysis to examine varied traffic flow profiles during the weekdays.

Fig. 10 shows the correlation between $R I 3_{\text {in }}$ and delay of J3a for two periods of time, October 2002 to April 2003 in Fig. 10a and October 2006 to April 2007 in Fig. 10b. Both $R I 3_{\text {in }}$ and delay were calculated as the average for the total period considered at $15 \mathrm{~min}$ intervals. $R I 3_{\text {in }}$ for J3a showed very strong correlation with the junction delay for both time periods as depicted from Fig. 10, confirming the results from the Delft case study.

Furthermore, Fig. 11 shows the variation of $R I 3_{\text {in }}$ for the two time periods, October 2002 to April 2003 (pre ATM activation) and October 2006 to April 2007 (after the activation of ATM scheme). Comparing $R I 3_{\text {in }}$ for the time period October 2002 to April 2003 with October 2006 to April 2007 shows that the scheme results in a general improvement in the redundancy index $R I 3_{\text {in }}$ as depicted from Fig. 11. The amount of improvement varies throughout the day, for example at 6:30 am (offpeak) both values are very similar, meanwhile there are noticeable improvements between 7:45 am to $11: 00 \mathrm{pm}$ with different rates.

Fig. 12 shows the impact of capacity increase by considering the period between January to April 2006 (3L-VMSL) and the period from January to April 2007 (4L-VMSL). A little improvement in $R I 3_{\text {in }}$ due to the use of the hard shoulder, especially the morning peak is observed. However, the ATM scheme has attracted more traffic flow (as shown in Fig. 13) for both periods that could negatively affected the improvement of $R I 3_{i n}$.
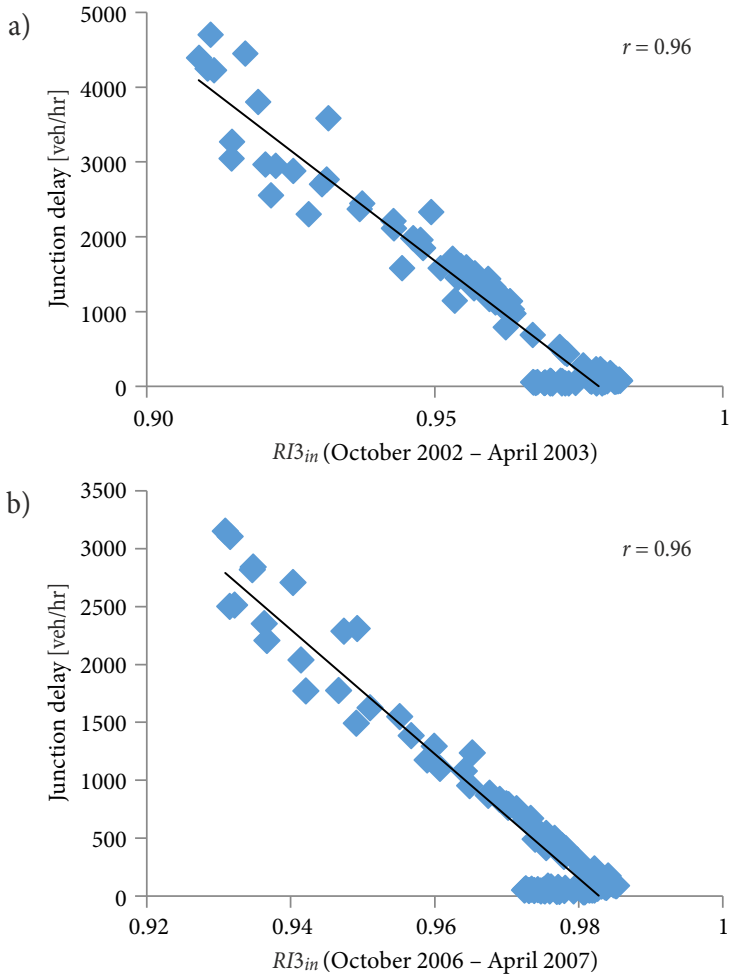

Fig. 10. $R I 3_{\text {in }}$ and total delay: a $-R I 3_{\text {in }}$ and junction delay (October 2002 to April 2003, No-VMSL); b - RI $3_{\text {in }}$ and junction delay (October 2006 to April 2007, 4L-VMSL)

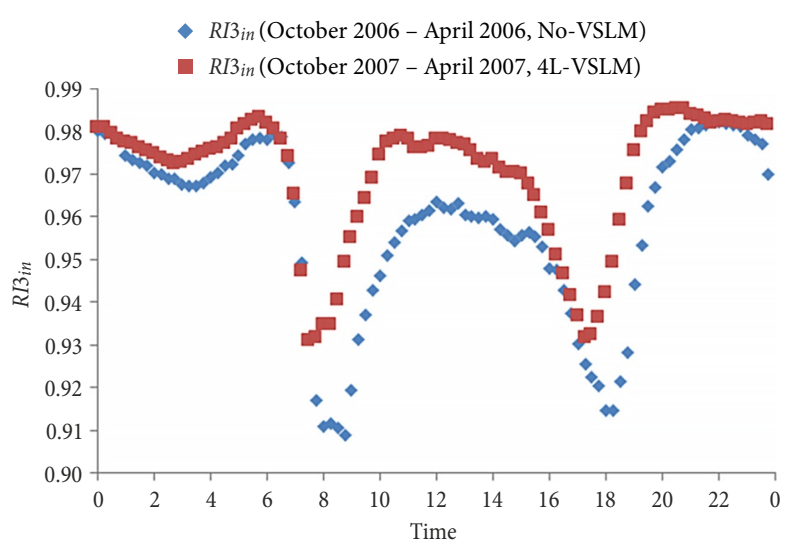

Fig. 11. $R I 3_{\text {in }}$ for the time periods October 2002 to April 2003 and October 2006 to April 2007

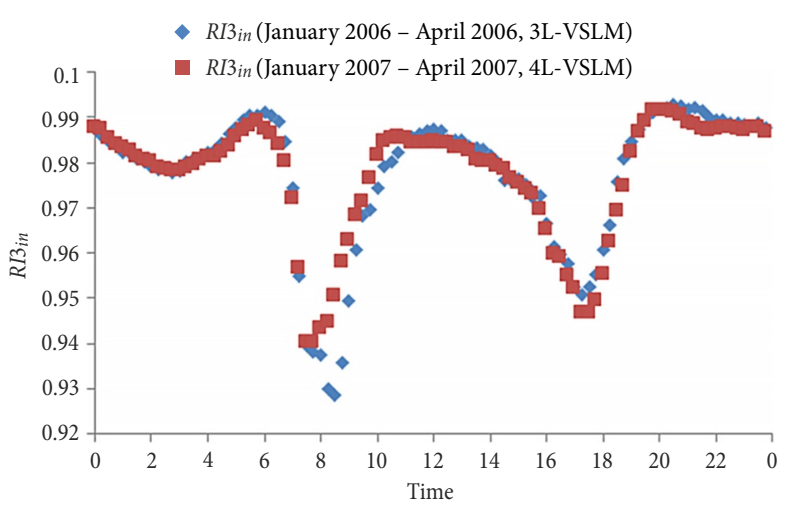

Fig. 12. $R I 3_{\text {in }}$ for the time periods January to April 2006 and January to April 2007 


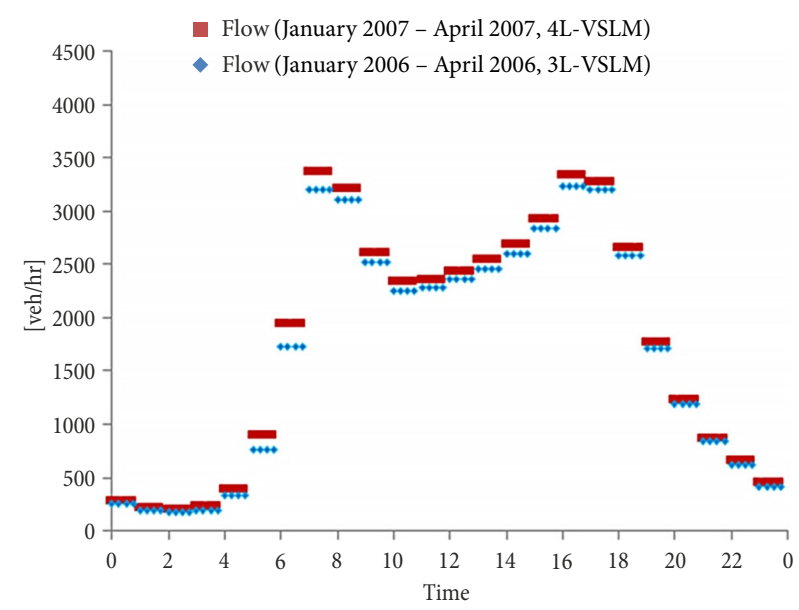

Fig. 13. Variation of traffic flow for the time periods January to April 2006 and January to April 2007

\section{Conclusions}

The main aim of this paper was to introduce a redundancy index for various nodes in road transport networks that is able to cover both static and dynamic aspects of redundancy. The static aspect of redundancy refers to the existence of alternative paths to a certain node whereas the dynamic aspect covers the issues related to the availability of spare capacity under different network loading and level of service such as the relative average speed. The proposed technique is based on the entropy concept owing to its ability to measure the configuration of a system in addition to being able to model the uncertainties inherent in road transport network. In contrast with previous investigations on redundancy in water systems based on one system characteristic, a number of redundancy indices were developed from combinations of link characteristics to enhance their correlations with the junction delay and the volume capacity ratio.

For each proposed redundancy index two values are calculated (i.e. outbound redundancy and inbound redundancy indices) to quantify the redundancy level of each node in the network. It was found that none of the outbound redundancy indices correlated well with the junction delay or junction volume capacity ratio. Consequently, the analysis focused on the inbound redundancy indices as they were able to reflect the variations in topology of the nodes (e.g. number of incident links) and the variation in link speed. However, further research is recommended to investigate the impact of the outbound links on the junction redundancy index. A network redundancy index is also developed by aggregating a weighted redundancy index for all the nodes.

Two case studies based on a synthetic road transport network of Delft city and Junction 3A in M42 motorway near Birmingham are considered to test the ability of the redundancy indices to reflect various network conditions and demand variation. Each proposed redundancy index was assessed against the junction delay and volume capacity ratio and consequently two redundancy indices based on combined relative link speed and relative link spare capacity were chosen. Furthermore, the suitability of each redundancy index relies on the junction type based on analysis of various junction types in the synthetic road transport network of Delft city. The two chosen redundancy indices responded well to the variation in demand under the same network conditions as well as supply variation, for example network capacity reduction.

The proposed redundancy indices could be a potential tool to identify the optimal design alternatives during the planning stage of the network junctions in addition to the best control and management policies under disruptive events or for daily operation of the road transport network.

\section{References}

Allesina, S.; Azzi, A.; Battini, D.; Regattieri, A. 2010. Performance measurement in supply chains: new network analysis and entropic indexes, International Journal of Production Research 48(8): 2297-2321. http://doi.org/10.1080/00207540802647327

Andreson, W. P.; Maoh, H.; Burke, C. 2011. Assessing risk and resilience for transportation infrastructure in Canada, in CTRF 46th Annual Conference - Transportation and Innovation: the Roles of Governments, Industry and Academia, 29 May - 1 June 2011, Quebec, Canada, 298-312.

Awumah, K.; Goulter, I.; Bhatt, S. K. 1991. Entropy-based redundancy measures in water-distribution networks, Journal of Hydraulic Engineering 117(5): 595-614.

http://doi.org/10.1061/(ASCE)0733-9429(1991)117:5(595)

Boccaletti, S.; Latora, V.; Moreno, Y.; Chavez, M.; Hwang, D.-U. 2006. Complex networks: structure and dynamics, Physics Reports 424(4-5): 175-308. http://doi.org/10.1016/j.physrep.2005.10.009

Corson, F. 2010. Fluctuations and redundancy in optimal transport networks, Physical Review Letters 104(4): 1-4. http://doi.org/10.1103/PhysRevLett.104.048703

DfT. 2011. Road Network Policy Consultation. Department for Transport (DfT), London, UK. 37 p. Available from Internet: www.gov.uk/government/uploads/system/uploads/attachment_data/file/2439/roadnetworkconsultation.pdf

Downer, J. 2009. When Failure is an Option: Redundancy, Reliability and Regulation in Complex Technical Systems. Discussion Paper No 53. Centre for Analysis of Risk and Regulation (CARR). London, UK. 27 p. Available from Internet: http://eprints.lse.ac.uk/36537/1/Disspaper53.pdf

El-Rashidy, R. A.; Grant-Muller, S. M. 2014. An assessment method for highway network vulnerability, Journal of Transport Geography 34: 34-43. http://doi.org/10.1016/j.jtrangeo.2013.10.017

Erath, A.; Löchl, M.; Axhausen, K. W. 2009. Graph-theoretical analysis of the Swiss road and railway networks over time, Networks and Spatial Economics 9(3): 379-400. http://doi.org/10.1007/s11067-008-9074-7

eTN. 2010. The Tourism Industry Implications of the Eyjafjallajokull Volcano Eruption. eTN Global Travel Industry News. Available from Internet: http://www.eturbonews. com/15558/tourism-industry-implications-eyjafjallajokullvolcano-eruption

Haimes, Y. Y. 2009. On the definition of resilience in systems, Risk Analysis 29(4): 498-501.

http://doi.org/10.1111/j.1539-6924.2009.01216.x 
Highways Agency. 2013. HATRIS JTDB Data Guide V7: status in January 2013. Highways Agency, UK.

Hoshiya, M.; Yamamoto, K. 2002. Redundancy index of lifeline systems, Journal of Engineering Mechanics 128(9): 961-968. http://doi.org/10.1061/(ASCE)0733-9399(2002)128:9(961)

Hoshiya, M.; Yamamoto, K.; Ohno, H. 2004. Redundancy index of lifelines for mitigation measures against seismic risk, Probabilistic Engineering Mechanics 19(3): 205-210. http://doi.org/10.1016/j.probengmech.2004.02.003

Hyder Consulting. 2010. Network Resilience and Adaptation. Final Report. UK.

Immers, B.; Stada, J.; Yperman, I.; Bleukx, A. 2004. Towards robust road network structures, Slovak Journal of Civil Engineering 12(4): 10-17.

Javanbarg, M. B.; Takada, S. 2007. Redundancy model for water supply systems under earthquake environments, in Proceedings of 5th International Conference on Seismology and Earthquake Engineering, May 2007, Tehran, Iran, 1-8.

Jenelius, E. 2010. Redundancy importance: links as rerouting alternatives during road network disruptions, Procedia Engineering 3: 129-137. http://doi.org/10.1016/j.proeng.2010.07.013

Jenelius, E. 2009. Network structure and travel patterns: explaining the geographical disparities of road network vulnerability, Journal of Transport Geography 17(3): 234-244. http://doi.org/10.1016/j.jtrangeo.2008.06.002

Kanno, Y.; Ben-Haim, Y. 2011. Redundancy and robustness, or when is redundancy redundant?, Journal of Structural Engineering 137(9): 935-945. http://doi.org/10.1061/(ASCE)ST.1943-541X.0000416

Koç, Y.; Warnier, M.; Kooij, R. E.; Brazier, F. M. T. 2013. An entropy-based metric to quantify the robustness of power grids against cascading failures, Safety Science 59: 126-134. http://doi.org/10.1016/j.ssci.2013.05.006

Lhomme, S.; Serre, D.; Diab, Y.; Laganier, R. 2013. Analyzing resilience of urban networks: a preliminary step towards more flood resilient cities, Natural Hazards and Earth System Sciences 13(2): 221-230.

http://doi.org/10.5194/nhess-13-221-2013

Miao, X.; Xi, B.; Guan, M.; Tang, Y.-H. 2011. Route entropy based capacity reliability assessment and application in multi-objective satisfactory optimization of logistics network, Scientific Research and Essays 6(16): 3335-3343. http://doi.org/10.5897/SRE10.1013

Mot, M. D. 2008. ATM Monitoring and Evaluation: 4-Lane Variable Mandatory Speed Limits - 12 Month Report (Primary and Secondary Indicators). Transport Research Laboratory, UK. 233 p.

Nagata, S.; Yamamoto, K. 2004. Strategy of water supply network restoration with redundancy index, in Proceedings of the 13 the World Conference on Earthquake Engineering (13 WCEE), 1-6 August 2004, Vancouver, BC, Canada, 1-11.

Ortúzar, J. D; Willumsen, L. G. 2011. Modelling Transport. 4 th edition. Wiley. $607 \mathrm{p}$.

Randles, M.; Lamb, D.; Odat, E.; Taleb-Bendiab, A. 2011. Distributed redundancy and robustness in complex systems, Journal of Computer and System Sciences 77(2): 293-304. http://doi.org/10.1016/j.jcss.2010.01.008

Rodrigue, J.-P.; Comtois, C.; Slack, B. 2013. The Geography of Transport Systems. 3rd edition. Routledge. $432 \mathrm{p}$.

Shannon, C. E. 1948a. A mathematical theory of communication, The Bell System Technical Journal 27(3): 379-423. http://doi.org/10.1002/j.1538-7305.1948.tb01338.x
Shannon, C. E. 1948b. A mathematical theory of communication, The Bell System Technical Journal 27(4): 623-656. http://doi.org/10.1002/j.1538-7305.1948.tb00917.x

Snelder, M.; Van Zuylen, H. J.; Immers, L. H. 2012. A framework for robustness analysis of road networks for short term variations in supply, Transportation Research Part A: Policy and Practice 46(5): 828-842.

http://doi.org/10.1016/j.tra.2012.02.007

Streeter, C. L. 1992. Redundancy in organizational systems, Social Service Review 66(1): 97-111. http://doi.org/10.1086/603897

Sultan, B.; Meekums, R.; Brown, M. 2008. The impact of active traffic management on motorway operation, in Road Transport Information and Control - RTIC 2008 and ITS United Kingdom Members' Conference, IET, 20-22 May 2008, Manchester, UK, 1-8. http://doi.org/10.1049/ic.2008.0793

Sun, L.; Rong, J.; Yao, L.; Xu, H.; Liu, H. 2012. Entropy-based estimation of transfers in a terminal, Transportation Planning and Technology 35(3): 303-315. http://doi.org/10.1080/03081060.2012.671038

Swanson, G. A.; Bailey, K. D.; Miller, J. G. 1997. Entropy, social entropy and money: a living systems theory perspective, Systems Research and Behavioral Science 14(1): 45-65. http://doi.org/10.1002/(SICI)1099-1743(199701/02)14: $1<45:: A I D-S R E S 151>3.0 . C O ; 2-Y$

Wardrop, J. G. 1952. Some theoretical aspects of road traffic research, Proceedings of the Institution of Civil Engineers 1(3): 325-362. http://doi.org/10.1680/ipeds.1952.11259

Wilson, A. G. 1970. The use of the concept of entropy in system modelling, Journal of the Operational Research Society 21(2): 247-265. http://doi.org/10.1057/jors.1970.48

Yazdani, A.; Jeffrey, P. 2012. Applying network theory to quantify the redundancy and structural robustness of water distribution systems, Journal of Water Resources Planning and Management 138(2): 153-161. http://doi.org/10.1061/(ASCE)WR.1943-5452.0000159 